Silvia Gilardoni

Università Cattolica del Sacro Cuore di Milano

https://doi.org/10.18778/8220-506-0.11

\title{
IL DESIGN ITALIANO E L'ITALIANITÀ NEI NOMI COMMERCIALI. PERCORSI PER LA DIDATTICA DELL'ITALIANO L2
}

Riassunto: Il lavoro prende in esame l'espressione dell'italianità nella creazione dei nomi commerciali nel settore del design italiano, al fine di individuare elementi linguistici e culturali da integrare in percorsi didattici per lo sviluppo della competenza linguistica e interculturale in apprendenti di italiano come lingua seconda o straniera.

La ricerca si basa sull'analisi linguistico-semantica di un corpus di nomi commerciali di aziende e prodotti del design italiano, che ha permesso di considerare il ruolo della creatività onomastica nella diffusione dell'italianità nel mondo. A tale analisi segue la riflessione glottodidattica, volta a delineare suggerimenti applicativi per la creazione di attività didattiche su nomi commerciali del design nella classe di italiano L2.

Parole chiave: onomastica commerciale, didattica dell'italiano L2, design italiano.

\begin{abstract}
Italian design and Italianity in brand naming. Teaching activities in Italian as a second language. This paper examines the expression of Italian language and culture in brand naming for the Italian design sector, in order to identify linguistic and cultural elements to be integrated into teaching activities for the development of linguistic and intercultural competence in Italian as a second language.

The research is based on the linguistic and semantic analysis of a corpus of brand names of Italian design companies and products, which has allowed us to consider the role of onomastic creativity in the spread of Italian language and culture in the world. The analysis is followed by some suggestions for planning teaching activities based on the use of Italian design brand names in the context of teaching and learning Italian as a second language.
\end{abstract}

Keywords: brand naming, teaching Italian as a second language, Italian design. 


\section{Introduzione}

In questo contributo prendiamo in considerazione l'espressione dell'italianità nei nomi commerciali in un ambito di eccellenza della realtà imprenditoriale italiana, il settore del design. Obiettivo del lavoro è l'individuazione di elementi linguistici e culturali che possano essere oggetto di percorsi didattici nel contesto dell'insegnamento/apprendimento dell'italiano L2.

A tale scopo, a partire dalla selezione di un campione rappresentativo di nomi commerciali di aziende e prodotti, è stata condotta un'analisi linguisticosemantica di marchionimi del settore del design italiano, al fine di esaminare la correlazione tra creatività onomastica e diffusione dell'italianità e avanzare proposte applicative nella prospettiva della didattica dell'italiano $\mathrm{L} 2$.

Dopo l'illustrazione del quadro teorico e metodologico in cui si colloca la ricerca $(\$ 2)$, viene presentata l'analisi del corpus di marchionimi raccolto $(\$ 3)$, cui seguono le riflessioni di carattere glottodidattico ( $\$ \$ 4-5)$.

\section{Il quadro teorico e metodologico}

La ricerca ha preso avvio dalla considerazione della centralità del concetto di italianità non solo nell'ambito delle analisi sulla diffusione dell'italiano nel mondo, ma anche in una prospettiva glottodidattica.

Le riflessioni linguistiche e storico-culturali sulla presenza e la forza di espansione dellalingua e cultura italiana nel mondo hanno evidentiimplicazioni, in primo luogo, nella definizione di strategie di intervento nel quadro di una politica linguistica che intenda promuovere l'identità italiana nel mondo. La nozione di italianità può essere associata così a quella di italicità, un concetto risemantizzato da Bassetti (Roić 2006; Bassetti 2010, 2015), poi ripreso da Vedovelli (Vedovelli 2011; Turchetta, Vedovelli 2018) e da Bombi e Orioles (Bombi, Orioles 2011, 2014; Orioles 2014, 2015), per identificare unampia “'sfera d'influenza' della lingua e cultura italiana nel mondo", non riconducibile solo all'appartenenza identitaria di carattere etnico-linguistico, come nel caso degli italo-discendenti, o di tipo giuridico, come nel caso dei cittadini italiani all'estero, ma estendibile a tutti quei soggetti, tra cui anche i migranti in Italia, "desiderosi $[\ldots]$ di entrare nell'orbita dello spazio culturale italiano, nella misura in cui si riconoscano in un sistema di valori e ne condividano i codici" (Bombi, Orioles 2014: 309).

La presenza dell'italianità nel mondo è strettamente correlata ai diversi elementi che concorrono alla diffusione dell'italiano, quali il vasto patrimonio della tradizione culturale italiana in campo artistico, letterario, musicale ecc., 
l'ampiezza dei movimenti migratori degli italiani nel mondo e degli stranieri in Italia e la ricchezza delle espressioni della società e del sistema produttivo italiano, come l'enogastronomia, la moda o il design.

Si tratta di quei fattori di attrattività della lingua e della cultura italiana, chiaramente evidenziati nella grande indagine Italiano 2000 sui pubblici dell'italiano e sulle motivazioni allo studio dell'italiano nel mondo (De Mauro et al. 2002), che risultano rilevanti anche in ambito glottodidattico: i diversi fattori di attrattività dell'italiano, infatti, sono all'origine di motivazioni e interessi degli apprendenti e costituiscono elementi centrali nella progettazione didattica, per la selezione di testi, funzioni comunicative, aspetti linguistici e culturali che rispondano ai bisogni della formazione in italiano L2.

In questo lavoro abbiamo voluto focalizzare l'attenzione sulla realtà italiana del design, che appartiene alla tradizione culturale del Paese, ma è anche altamente rappresentativa di quel settore di creatività e produttività economica identificato nel cosiddetto made in Italy, cui si lega oggi, in particolare, la "diffusione e il prestigio internazionale" dell'italiano (Bombi, Orioles 2011:22).

Il design, o più estesamente l'industrial design, in italiano il "disegno industriale", ha origine nel periodo della Rivoluzione industriale e, più precisamente, in Inghilterra, nel momento in cui si assiste al passaggio nella realizzazione dell'oggetto "dalla dimensione delle botteghe artigiane, dal 'fatto a mano' in genere in pezzo unico, a quello della sua riproduzione seriale" (Vercelloni 2014: 11).

In Italia dalla seconda metà dell'Ottocento comincia a diffondersi una nuova cultura tecnica e applicata, grazie alla nascita di scuole di specializzazione, dei Politecnici, di scuole di arti e mestieri e scuole di arte applicata all'industria e, con l'inizio del Novecento, vengono promosse fiere ed esposizioni. Si giunge così alla progressiva affermazione del design italiano, con le mostre internazionali biennali delle arti decorative a Monza, inaugurate nel 1923, e le mostre triennali di Milano a partire dal 1933, fino alla nascita del "Bel Design italiano" nel periodo della ricostruzione del secondo dopoguerra: si tratta di "una sorta di complessa dimensione progettuale", come ricorda Vercelloni (2014: 90), “in grado di apportare all'oggetto d'uso, all'arredo e alle attrezzature per la casa, ma anche ai mezzi di locomozione e trasporto, quella sorta di surplus estetico-formale e d'invenzione tipologica che ha reso famoso il design italiano nel mondo".

Nasce quindi il fenomeno delle cosiddette "Fabbriche del design italiano", una serie di aziende che hanno contribuito alla storia e alla diffusione del design made in Italy, come viene sintetizzato da Alessi (2014: 13-14):

Con Fabbriche del Design Italiano intendiamo una ventina di aziende del settore che si sono impresse nella memoria della critica e degli studiosi, nonché dei loro imprenditori, e che convenzionalmente sono accomunate 
da alcune costanti: le dimensioni medio-piccole, il fatto di avere qualche decennio di vita alle spalle e di essere solitamente a conduzione familiare, di aver sede in Italia (generalmente Milano e dintorni), di rivendicare una produzione interna alla propria sede e di poter vantare una genealogia di collezioni attivate in collaborazione con i principali Maestri del design italiano. Storicamente queste aziende hanno rappresentato per il mondo della cultura del progetto, anche oltre confine e oltre oceano, un riferimento di carattere imprescindibile e una stella polare tra gli esempi di imprenditoria italiana. [...]. Vanno dalla A di Artemide alla Z di Zanotta, passando per Alessi, Alias, B\&B, Brionvega, Cappellini, Cassina, Danese, De Padova, Driade, Edra, Flos, Flou, FontanaArte, Kartell, Magis, Meritalia, Moroso, Olivetti, Poliform, Poltrona Frau, Richard Ginori, Tecno, Venini, ecc.

All'indiscutibile riconoscimento internazionale dell'italianità del design non è seguita però, come osservano Coveri et al. (2016), una espansione della lingua italiana del design all'estero, diversamente da quanto si evidenzia per altri settori di eccellenza come, per esempio, l'enogastronomia o la moda, per i quali l'italiano vanta una significativa influenza su altre lingue e un'ampia presenza nei paesaggi linguistici delle città nel mondo ${ }^{1}$. Se il termine "design" rimanda all'italiano "disegno", passato all'inglese per tramite del francese, "per il prestigio dell'arte rinascimentale e della sua trattatistica” (Cartago 2016: 7), nel settore del design "a livello generale $[\ldots]$ non si registrano italianismi che siano riusciti a imporsi a livello internazionale"; lo stesso design italiano, pur nel delinearsi e consolidarsi di una lingua italiana settoriale del design, "ha sempre parlato inglese sin dalle origini" (Coveri et al. 2016: 23-24)2 . Coveri et al. (2016: 24) sottolineano, tuttavia, come possa rivelarsi interessante osservare le modalità di creazione dei nomi dei prodotti, in cui si riscontra l'uso dell'italiano anche in nomi "divertenti e ironici".

L'attribuzione di un nome proprio a un prodotto di design è legata alla scelta del designer, il progettista dell'oggetto, o a una decisione del produttore o del responsabile marketing dell'azienda ed è da intendersi come una "strategia identitaria" finalizzata a rendere l'oggetto "percepibile, riconoscibile e classificabile”, come osserva Annicchiarico (2002: 22). Ideatrice e curatrice di un ciclo di mostre dal titolo Le parole e le cose, realizzato nel corso del 2002 alla Triennale di Milano, Annicchiarico, esplorando il

1 Sull'influenza della lingua italiana nell'ambito enogastronomico, rimandiamo, tra gli altri, a Frosini (2012) e Bombi (2014); in riferimento al settore della moda si veda ad esempio Sergio (2016). Sulla presenza dell'italiano nei panorami linguistici urbani cfr. Vedovelli (2005) e Bagna, Barni (2007).

2 Per una storia della lingua italiana del design si veda Dellapiana, Siekiera $(2015,2017)$. 
rapporto tra i nomi e gli oggetti del design, sottolinea come il nome sia parte del progetto stesso che genera l'oggetto. Grazie al nome l'oggetto diventa "un'entità sociale", ossia "un artefatto individuabile, interpretabile, vendibile e comprabile nel commercio (anche simbolico) e nelle pratiche di scambio della comunità che decide di usarlo, di farlo proprio e di conferirgli un valore"; in tal modo il nome contribuisce alla "narrativizzazione dell'oggetto" che lo porta, comunicando qualcosa sull'oggetto, sulla sua origine, le sue caratteristiche o il suo utilizzo, e suscitando associazioni ed evocazioni semantiche (Annicchiarico 2002: 23-26).

Il processo di assegnazione dei nomi agli oggetti di design è frutto, tuttavia, di un percorso evolutivo. Agli inizi della storia del design italiano gli oggetti venivano identificati con nomi comuni descrittivi, come i "servizi in porcellana da camera" di Richard Ginori del 1905, oppure con codici fatti di sigle e sequenze di numeri, come nel caso delle prime macchine da scrivere di Olivetti, la M1 del 1911 e la M20 del 1920. Nel periodo tra le due guerre le strategie di denominazione dei prodotti cominciano a cambiare; si possono ricordare per esempio la Balilla del 1932 e la Topolino del 1943 di FIAT nel settore dei mezzi di trasporto, oppure le macchine da scrivere Auctor (1932) e Studio (1935) o la macchina da calcolo Summa (1940) di Olivetti (Annicchiarico 2002: 24; Papa 2015: 774-779). Con il secondo dopoguerra e in particolare negli anni Cinquanta del boom economico le aziende iniziano poi a porsi "in modo sistematico il problema del nome da dare agli oggetti", prima con una finalità "funzionale", poi "sempre più in modo emozionale" (Annicchiarico 2002: 24-25).

Se pur continua la possibilità di denominare i prodotti con semplici codici identificativi, nel caso in cui il designer o il produttore non abbiano mostrato interesse per la creazione di un nome, le strategie di naming si evolvono e si diversificano: emerge la necessità da parte di "chi progetta, disegna e costruisce", come testimonia l'architetto e designer Piero Lissoni, "di trovare un vocabolo giusto, originale, mai sentito, efficace, musicale, evocativo, sensuale, cioè desiderabile"

Come viene riportato dalla voce di Alberto Alessi, attualmente alla direzione dell'azienda Alessi, una tra le più note "Fabbriche del design italiano", nella creazione dei nomi varia anche l'uso delle lingue: si passa dall'italiano all'uso di altre lingue, in particolare l'inglese (Coveri et al. 2016).

Abbiamo voluto indagare, quindi, tale creatività onomastica, nella prospettiva di un'analisi linguistica e semantica dei nomi commerciali, secondo

3 Riportiamo un estratto di un'intervista tratta dall'articolo di F. Gugliotta, Le cose e i loro nomi, "Casa\&Design", 18 maggio 2018, design.repubblica.it/2018/05/18/ le-cose-e-i-loro-nomi/\#1 [20/05/2020]. 
la metodologia proposta da Cotticelli Kurras (2008, 2011, 2012), al fine di rilevare la manifestazione dell'italianità nel settore del design italiano.

Dal punto di vista glottodidattico abbiamo accolto poi i suggerimenti metodologici di Gałkowski, che considera la conoscenza dei nomi commerciali come un elemento della "competenza linguistica e interculturale" degli apprendenti (Gałkowski 2011: 79). I nomi commerciali o marchionimi, in quanto sottocategoria dei nomi propri, sono parte di quella "competenza onomastica" che contribuisce alla conoscenza e alla comprensione della cultura di un Paese e può essere sfruttata in modo creativo nella didattica (Gałkowski $2011,2015)^{4}$. Lo studioso si richiama al concetto di "lessicultura" proposto da Galisson (1988), che sottolinea la rilevanza perl'insegnamento/apprendimento delle lingue e delle culture di quelle parole con un valore culturale condiviso ("mots à charge culturelle partagée") in una comunità in un dato momento, tra cui si collocano anche i marchionimi (Gałkowski 2011: 89-90)5.

In tal senso i nomi degli oggetti del design italiano, insieme ai nomi delle aziende produttrici, sono parte della storia e della realtà culturale e sociale italiana e possono essere utilizzati per la realizzazione di attività e la progettazione di percorsi per la didattica dell'italiano L2.

\section{Nomi commerciali nel settore del design e italianità: analisi di un corpus}

Per indagare le modalità di espressione dell'italianità nei nomi commerciali del mondo del design, data l'ampiezza dei dati esistenti, abbiamo orientato la ricerca verso l'individuazione di un campione di casi esemplari e significativi delle strategie di naming del settore; abbiamo così creato un corpus di nomi di prodotti e delle relative aziende produttrici, a partire dagli oggetti esposti al Museo del Design Italiano presso la Triennale di Milano, un allestimento permanente, inaugurato nel 2019, che presenta una selezione dei pezzi più iconici del design italiano, molti dei quali sono esposti anche al MOMA - The Museum of Modern Art di New York.

4 Rimandiamo, come suggerisce anche Gałkowski, alla definizione terminologica proposta da Caffarelli (2011) di "nome commerciale" o "marchionimo", inteso come "il nome con cui è noto in commercio un determinato prodotto o il nome dell'azienda che lo produce". Per Gałkowski tali nomi rientrano nella più ampia tipologia dei crematonimi, nomi usati per denominare cose prodotte dall'uomo di tipo materiale o immateriale, e sono compresi nei crematonimi del marketing (Gałkowski 2010, 2016).

5 A Galisson si deve anche la redazione, in collaborazione con André, di un dizionario di nomi commerciali francesi (Galisson, André 1998). 
Si tratta di 176 nomi di prodotti e 71 nomi di aziende, rappresentativi di oltre trent'anni di storia del design italiano, dal 1946 al $1981^{6}$.

I nomi raccolti sono stati analizzati e classificati in base alla lingua utilizzata, alla struttura morfologica e alle associazioni semantiche cui rimandano (Cotticelli Kurras 2008, 2011, 2012).

Per ricostruire l'origine e i significati dei nomi sono state consultate diverse fonti: le informazioni relative ai marchionimi aziendali sono state recuperate attraverso i siti delle aziende e alcuni archivi storici online ${ }^{7}$; per l'origine dei nomi degli oggetti abbiamo consultato cataloghi di mostre, archivi e testi di maestri del design italiano, studi di esperti del settore, riviste e blog specializzati, oltre ai siti aziendali ${ }^{8}$.

\subsection{I nomi aziendali}

Tra i nomi aziendali prevale decisamente l'uso della lingua italiana (83\%). La maggior parte dei marchionimi (63\%) rimandano infatti ai nomi italiani dei fondatori delle aziende. Sono in genere utilizzati i soli antroponimi, come ad esempio nel caso di Alessi, Boff, Cappellini, Cassina, Necchi, Olivetti, Sambonet o Zanotta, che possono però occorrere anche in combinazioni sintagmatiche di vario tipo: è il caso del sintagma Fratelli Guzzini oppure di altre combinazioni come Danese Milano, in cui al nome del fondatore si accosta il luogo della

6 Per la selezione degli oggetti, con le indicazioni dei relativi nomi e dei produttori, abbiamo fatto riferimento al sito della Triennale, in particolare alla pagina dedicata al Museo del Design Italiano (www.triennale.org/risultati-archivio?archiveId=collezioni \&archives=permanente\&freeText=\&isPresentInMuseum=true [20/05/2020]).

7 Oltre ai siti delle aziende, abbiamo consultato il sito del Museo del marchio italiano e l'archivio della Fondazione Fiera Milano (www.museodelmarchioitaliano. it/, archiviostorico.fondazionefiera.it/ [20/05/2020]).

8 Abbiamo fatto riferimento ai cataloghi delle mostre curate da Annicchiarico per la Triennale di Milano (2002a, 2002b, 2002c, 2007), insieme ad alcuni siti dedicati ai designer italiani Achille Castiglioni, Giorgina Castiglioni, Ugo La Pietra, Vico Magistretti, Federica Marangoni e Aldo Rossi (fondazioneachillecastiglioni.it/, www. giorginacastiglioni.it/, ugolapietra.com/, archivio.vicomagistretti.it/magistretti/, www.federicamarangoni.com/, www.fondazionealdorossi.org/ [20/05/2020]).

Abbiamo poi consultato un volume sulla figura del designer Vittoriano Viganò (Piva, Cao 2009), un saggio sui nomi delle macchine di Olivetti (Papa 2015), un volume sull'evoluzione delle geometrie e dell'uso dei materiali nel design (Sala, Sala 2005) e uno studio sulla storia della pulizia industriale (Guizzi 2015). Alcune informazioni sono state reperite anche sulle riviste specializzate "Domus" e "Casa\&Design" (www.domusweb.it/it/design.html, design.repubblica.it/ [20/05/2020]) e sui blog "Sbandiu. Momenti di design" e "Moobilia" (sbandiu.com/; www.moobilia.it/ magazine/ [20/05/2020]). 
sede, oppure ancora Fontana Arte, Martinelli Luce e Poltrona Frau, in cui l'antroponimo è unito a un nome comune che rimanda ad aspetti e tipologie della produzione. Talvolta i nomi dei fondatori sono parte di acronimi o sigle, come ad esempio nel caso di Gufram (Gugliermetto Fratelli Arredamenti Moderni), oppure occorrono in neoformazioni, come nel caso del marchio Brionvega, legato all'imprenditore Giuseppe Brion già proprietario della ditta Radio Vega Television. Anche il marchionimo Kartell potrebbe rientrare in questa tipologia, trattandosi probabilmente di un gioco di parole sui cognomi dei due soci fondatori, Castelli e Rastelli, con resa straniera nella grafia.

Acronimi e neoformazioni sono utilizzati anche per altri riferimenti semantici. Ricordiamo ad esempio l'acronimo F.I.L.A. che sta per Fabbrica Italiana Lapis ed Affini e alcuni casi di neoformazioni come l'uso del prefissoide Tecno o le forme univerbate Arredoluce e Arteluce.

Si rileva poi l'uso di nomi comuni, come nei seguenti marchionimi: il marchio delle penne Aurora, che richiama all'idea di rinascita nel periodo del primo dopoguerra in cui si colloca la fondazione dell'azienda $(1919)^{9}$; il nome dell'azienda Tecnica, che richiama alla centralità della ricerca e dell'innovazione tecnologica che caratterizza la produzione dell'azienda nel settore dell'equipaggiamento sportivo; il marchio della ditta Vortice, dal nome del primo aspiratore per cappe da cucina creato nel 1954 da Attilio Pagani, il fondatore dell'azienda.

Si riscontra anche l'uso di nomi di origine mitologica come i noti Artemide e Driade, con cui si evocano valori della classicità e antichi saperi, che vengono a unirsi a sperimentazioni estetiche e ricerche tecnologiche innovative.

In pochi casi si osserva il ricorso ad altre lingue, tra cui il latino, l'inglese e il tedesco. Tra i nomi in latino ricordiamo i marchi Rex e Flos: il marchio dell'azienda di elettrodomestici Rex prende il nome dall'omonimo transatlantico italiano che nel 1933 conquistò il "Nastro Azzurro" nell'attraversamento dell'Atlantico da Genova a New York; Flos è il nome scelto per l'azienda da Pier Giacomo Castiglioni, per richiamare con il concetto di "fiore" un "pensiero luminoso", l' "idea di far nascere da una lampadina oggetti adatti a cambiare il modo di abitare", come si legge sul sito aziendale. In lingua inglese citiamo Memphis, il cui nome riprende un preciso evento che ha avuto come protagonista il designer Ettore Sottsass e che viene così ricordato sul sito dell'azienda: "È la sera dell'11 dicembre 1980. Nel soggiorno di Ettore Sottsass è riunito un gruppo di giovani designer e architetti. Un disco suona ripetutamente il brano 'Stuck Inside of Mobile with the Memphis Blues Again' di Bob Dylan: nasce

9 Ringraziamo Cesare Verona, presidente e amministratore delegato di Aurora ed esponente della quarta generazione della famiglia alla guida dell’azienda, per le informazioni fornite sulla storia del nome del marchio. 
Memphis, con duplice riferimento all'antica capitale dei faraoni egizi e alla città natale di Aretha Franklin ed Elvis Presley in Tennessee". Ricordiamo poi il caso di Lorenz, fondata nel 1934 da Tullio Bolletta, che scelse per la sua azienda un nome di origine tedesca, ispirandosi all'idea di forza e solidità evocata dalla società metalmeccanica Ansaldo Lorenz, ma anche riprendendo il nome di un famoso negozio di orologi di Berlino, dove fu maestro di orologeria ${ }^{10}$.

Non mancano infine casi di ibridi linguistici (9\%), in cui si osserva la compresenza di lingue diverse, come per esempio: $B$ Line, in cui il termine inglese Line è preceduto dall'iniziale del fondatore Giorgio Bordin; Lips Vago, azienda nata nel 1911 dalla fusione della ditta milanese della famiglia Vago con la società della famiglia olandese Lips; alcune neoformazioni, come gli incroci di lessemi Poltronova, dal nome "poltrona" unito all'aggettivo latino "nova", e Arflex, dal sintagma "arredamenti flessibili", in cui l'aggettivo è reso in una versione anglicizzata.

\subsection{I nomi dei prodotti}

Analizzando i nomi dei prodotti raccolti, si è riscontrato che per la maggior parte degli oggetti è stata scelta l'attribuzione di un nome specifico, mentre solo il $10 \%$ è identificato con un codice composto da numeri e sigle.

Le denominazioni dei prodotti sono prevalentemente in lingua italiana $(60 \%)$, che viene utilizzata per la maggior parte per nomi creativi ed evocativi e in minima parte (10\%) per la semplice descrizione dell'oggetto, come nel caso di un Tavolo con ruote o di un Portariviste a quattro tasche.

Tra i nomi creativi in italiano, dal punto di vista morfologico, prevalgono formazioni da materiale linguistico esistente, composto soprattutto da nomi comuni (42\%) e combinazioni sintagmatiche (19\%), cui seguono in misura minore nomi propri di persona e nomi mitologici, nomi geografici e aggettivi; sono presenti anche neoformazioni (16\%) realizzate con varie tecniche, come prefissazione e suffissazione, composizione, incrocio di lessemi o giustapposizione.

Per la creazione dei nomi si riscontra anche l'uso dell'inglese (15\%), seguito dal riferimento ad altre lingue, che ricorrono in nomi geografici e nomi propri o in casi isolati di nomi comuni, con espressioni di forma greca, latina, francese e giapponese; alcuni nomi (9\%) sono formazioni ibride, caratterizzate dall'uso di italiano e inglese. La presenza di altre lingue, soprattutto l'inglese, mostra la collaborazione delle aziende con designer internazionali ma anche la forte internazionalizzazione del settore del design italiano.

10 Ringraziamo Pietro Bolletta, direttore generale di Lorenz e nipote di Tullio Bolletta, per le informazioni fornite sulla storia del nome del marchio. 
Dal punto di vista semantico sono emersi diversi tipi di riferimenti e motivazioni veicolati dai nomi degli oggetti.

La motivazione semantica che occorre più frequentemente è quella tra il nome e la forma o elementi formali dell'oggetto. Alcuni nomi si riferiscono alla forma in modo descrittivo e referenziale, come i nomi comuni del portacenere Cubo e delle lampade Arco e Lampadina o come la formazione creata per giustapposizione della lampada Cuboluce ${ }^{11}$.

Una serie di nomi di oggetti esprime, invece, motivazioni semanticamente meno trasparenti, costruite sulla base di relazioni di somiglianza e analogia con la forma o elementi dell'oggetto.

Tra questi nomi si ritrovano in primo luogo nomi di animali, come per esempio: la lampada Cobra, che grazie a un braccio girevole assume "le più tipiche posizioni del rettile che le dà il nome", quando si presenta "con il capo eretto e pronto allattacco" oppure quando "striscia sinuoso con il capo proteso in avanti" (Annicchiarico 2002b: 49); la lampada Pipistrello, che "allude attraverso la forma del diffusore in metacrilato bianco all'animale notturno alato" (Annicchiarico 2002b: 75); la sedia Canguro, che sembra richiamare a un canguro per "lo slancio delle gambe (disegnate come se fossero in procinto di compiere un salto)" e per "la sacca 'marsupiale' che si colloca nella tradizionale posizione della seduta" (Annicchiarico 2002b: 53); la lampada creata da Isao Hosoe con il nome giapponese Hebi, che significa "serpente", in quanto, "grazie alla curvabilità del tubo flessibile" con cui è realizzato il basamento, "può assumere movenze simili a quelle di un serpente" (Annicchiarico 2002b: 52); la poltrona Papillon, che si richiama al nome francese di "farfalla", per "l'esplicito principio compositivo e progettuale" della poltrona basato sul "rapporto simmetrico che lega le due ali di una farfalla" (Annicchiarico 2002b: 77); la lampada Boalum che, come suggerisce il neologismo "è una sorta di serpente luminoso" (Annicchiarico 2002b: 50).

Il richiamo alla forma dell'oggetto si verifica anche attraverso il ricorso a elementi lessicali appartenenti ad altri campi semantici di vario genere. Ricordiamo ad esempio i seguenti nomi e sintagmi: Pratone, una poltrona composta da lunghi steli verdi, che vogliono rappresentare il prato di un giardino; Sacco, un pouf, realizzato come un sacco riempito di pallini di polistirolo, che diventa una seduta imbottita; Capitello, una poltrona che imita nella forma un capitello ionico; Atollo, una lampada che con le sue forme geometriche, un cono su un cilindro e sopra a tutto una semisfera, richiama la forma di un atollo; Fiocco, una poltrona con una struttura in tubolare curvato, rivestita con una calza in tessuto elastico, con una forma che assomiglia al

11 Per le immagini degli oggetti citati, così come per le specificazioni degli autori e delle date di creazione dei prodotti, si rimanda al sito del Museo del Design Italiano. 
fiocco di un nastro; Donna, una poltrona "dalle linee sinuose e sensuali che nel loro palese antropomorfismo alludono a una dea preistorica della fertilità e richiamano la prosperosità del corpo femminile" (Annicchiarico 2002a: 38); Joe, una poltrona a forma di un gigantesco guanto da baseball, il cui nome è un omaggio al grande campione del baseball americano Joe Di Maggio; I sassi, pouf ed elementi ornamentali a forma di sassi; Cabina dell'Elba, un armadio che riproduce una cabina posta sulla spiaggia, come le cabine osservate e disegnate dal creatore Aldo Rossi durante un periodo passato all'Isola d'Elba; Tramonto a New York, un divano composto da un cuscino a forma di sole che poggia su una serie di cuscini a forma di grattacielo, che compongono lo skyline della città, inizialmente denominato da Gaetano Pesce Tramonto di New York per esprimere il timore del declino della città, ma poi ribattezzato da Cassina per suggerire una visione meno negativa (Annicchiarico 2002c: 65). Tra le neoformazioni che richiamano la forma dell'oggetto segnaliamo le seguenti: il prefissato Superonda, "uno dei primi divani senza scheletro, un blocco di poliuretano tagliato in due da un'onda", come si legge sul sito di Poltronova, e il composto Rossocactus, la versione cromatica rossa dell'appendiabiti Cactus, che ripropone la forma di un cactus a grandezza umana.

Un altro tipo di motivazione semantica ricorrente rimanda alla funzione che l'oggetto ricopre nella vita quotidiana e al suo uso.

I nomi possono riferirsi alla funzione del prodotto con una modalità descrittiva, che esprime direttamente un richiamo all'uso dell'oggetto, in modo più o meno trasparente, come nei seguenti esempi: il nome Abitacolo per una struttura abitabile per bambini; i nomi di alcuni prodotti storici di Olivetti, quali la macchina da scrivere Lettera 22, con cui si vuole indicare il numero di tutte le lettere necessarie per comporre le parole, la calcolatrice Divisumma, il cui neologismo latineggiante rimanda alla capacità di eseguire somme e divisioni, e la calcolatrice Logos, che riprende l'accezione di "calcolo" della parola greca (Papa 2015: 779-784); i sintagmi Pranzo in piedi (nome di un servizio da tavola per snack-bar), Il posto dei giochi (un paravento giocattolo per bambini) e Mini Kitchen (un monoblocco su rotelle con la funzione di una cucina mobile); il composto Tratto pen, una "penna-pennarello" con una punta sintetica che permette l'erogazione continua di inchiostro; il neologismo coniato per il modello di aspirapolvere Spalter, un incrocio di lessemi interpretabile come "spalatore di terra" (Guizzi 2015), ma anche come "spalla-terra", dato che l'apparecchio può essere utilizzato portandolo a spalla oppure appoggiandolo sul pavimento; il neologismo Ariante, creato per suffissazione ("aria" + suffisso "ante"), dato a un ventilatore; il latinismo Luminator, utilizzato dai fratelli Achille e Pier Giacomo Castiglioni nel 1955 per la creazione di una lampada da terra in omaggio alla lampada omonima di Pietro Chiesa del 1933. 
Per alcuni nomi il riferimento agli aspetti funzionali e all'uso del prodotto è richiamato in modo più indiretto, per rimandi di carattere metaforico, come nei seguenti casi: la sedia Universale, denominata con un aggettivo che evoca "l'adattabilità a ogni tipo di ambiente" (Annicchiarico 2007); la poltrona da ufficio Vertebra, il primo "modello esemplare di design ergonomico" che nel nome rimanda alla stretta relazione tra la seduta e la schiena della persona (Annicchiarico 2007); Carimate, che indica per metonimia la sedia disegnata da Vico Magistretti per la Club House del Golf Club della città di Carimate (Como); il divano letto Anfibio, il cui nome richiama per somiglianza la trasformabilità dell'oggetto che può essere due cose insieme, un divano e un letto.

Unaltra tecnica di naming comprende nomi che rimandano a un elemento funzionale specifico della progettazione e della struttura dell'oggetto oppure nomi che sono ideati per esaltare una caratteristica distintiva dell'oggetto. Come esempi di oggetti i cui nomi si riferiscono ad elementi strutturali e progettuali del prodotto riportiamo i seguenti: la sedia Tre pezzi "realizzata con soli tre 'pezzi' di legno (una seduta e due bracciolo/gambe) legati da otto bulloni" (Piva, Cao 2009: 94); il portacenere Spirale di Achille Castiglioni, costituito da "una boccia di acciaio" che "accoglie sul bordo una molla a spirale di acciaio che trattiene la sigaretta, impedendole di cadere, ed è facilmente asportabile per la pulizia del contenitore", come viene riportato dall'archivio della Fondazione Achille Castiglioni; lo sgabello Mezzadro, il cui nome è in relazione di contiguità con l'elemento utilizzato per realizzare il prodotto, ossia il sedile di un trattore.

Tra le denominazioni che richiamano una caratteristica peculiare del prodotto abbiamo riscontrato solo un caso di un nome altamente descrittivo, rappresentato dall'aggettivo Superleggera, la sedia di Gio Ponti, che "fa della leggerezza - al contempo - un vanto, una conquista e uno stile" (Annicchiarico 2007). Nella maggiore parte dei casi, invece, si rilevano associazioni evocative, come nei seguenti esempi: la lampada Eclisse, in cui l'intensità della luce è regolata "attraverso il suo paralume interno rotante che 'eclissa' la sorgente luminosa”, come si legge nel sito dell'azienda Artemide; il telefono Grillo, con "un trillo originale da cui prende il nome l'oggetto" (Annicchiarico 2002b: 48); il tavolo della serie di mobili Quaderna, caratterizzata, come si precisa sul sito aziendale, da "forme geometriche regolari rivestite di laminato bianco quadrettato", così da formare un "unico piano quadrettato 'con gambe", con un richiamo alla pagina di un quaderno evocato dal neologismo; la poltrona Blow e la sedia Plia, i cui nomi richiamano la peculiarità del prodotto, con una parola inglese per la poltrona gonfiabile Blow e con una neoformazione su base francese per la sedia pieghevole Plia; la Spaghetti Chair, in cui la seduta e lo schienale sono realizzati avvolgendo attorno al telaio tondini in pvc, che richiamano nella forma e nella caratteristica di rigidità ed elasticità il cibo italiano degli spaghetti, duri quando sono crudi e morbidi quando sono cotti, così come gli "spaghetti 
di gomma" della sedia, rigidi quando sono vuoti e morbidi ed elastici quando vengono usati (Annicchiarico 2007).

Un'altra strategia per denominare gli oggetti consiste nell'utilizzare nomi che si riferiscono, attraverso associazioni di carattere metaforico, all'idea creativa del prodotto e a una indicazione di stile. Ricordiamo ad esempio: Lady, la poltrona di Marco Zanuso che "richiama nell'eleganza delle linee, dei colori e dei materiali un'idea di raffinatezza e di 'nobiltà' che il nome esplicita ed accentua" (Annicchiarico 2002a: 28); Mirella, la macchina da cucire realizzata da Necchi nel 1957, con un nome femminile molto diffuso in quell'epoca, scelto per lanciare "una macchina da cucire semplice ed economica che ambiva a macchinizzare il lavoro domestico femminile", esorcizzando ed estetizzando la fatica del lavoro (Annicchiarico 2002a: 53); Valentine, la macchina da scrivere ideata dal designer Ettore Sottsass nel 1969, con un nome che allude all'eroina dei fumetti di Guido Crepax, per veicolare valori culturali innovativi e trasgressivi "rispetto alla linea classica e tradizionale creata in precedenza da Olivetti” (Annicchiarico 2002a: 92); Kyoto, un tavolo creato da Gianfranco Frattini dopo un viaggio in Giappone per studiare l'opera dei maestri locali e "ispirato dalle tecniche di lavorazione e dall'estetica di quei luoghi", come viene precisato sul sito aziendale; Tizio, una lampada che si presenta come un "oggetto nuovo, quasi surreale" senza "alcun riferimento con qualsiasi fonte luminosa precedente", come suggerisce il nome che Sapper gli ha attribuito (Sala, Sala 2005: 210); Toio, la lampada da terra dei fratelli Castiglioni, che deve il suo nome alla trasformazione ironica della parola inglese toy, essendo "composta dal giocoso assemblaggio di un faro di automobile e di uno stelo telescopico ispirato a una canna da pesca" e assumendo così "la magia di un giocattolo per il mondo degli adulti”, come si legge sul sito dell'azienda Flos; Moon boot, le calzature dopo sci create da Giancarlo Zanatta, con un marchio, creato per giustapposizione dei lessemi di lingua inglese moon e boot, ispirato "dal mitico sbarco sulla Luna di Neil Armostrong e dell'Apollo 11", come ricorda il sito aziendale di Tecnica.

Alcuni nomi rimandano, invece, a ricordi legati alla vita personale del designer o all'oggetto stesso, come ad esempio: la poltrona Luisa, il cui nome "è una dedica alla signora Luisa Colombini [...] per oltre vent’anni segretaria personale di Franco Albini” (Annicchiarico 2002: 50); la poltrona Mies del 1969, il cui nome "evidenzia l’analogia della sua struttura con i progetti di Mies van der Rohe e rende omaggio al grande maestro deceduto nel medesimo anno", come viene riportato sul sito di Poltronova; la poltrona Sanluca, progettata dai fratelli Castiglioni per Dino Gavina nel 1960, che riprende nel nome l'omonimo portico di Bologna dove è stata fotografata.

Alcuni nomi, infine, sono legati a scelte operate dall'azienda produttrice, come nel caso di Artemide, Danese e Memphis. Artemide, come ricorda 
Annicchiarico (2002: 29), "dopo aver assunto il nome della dea della luce, per molti anni decide di chiamare tutte le lampade prodotte, a cascata, con nomi mitologici"; tra queste il Museo del Design Italiano ospita la lampada da tavolo con il nome del centauro Nesso, cui si aggiunge la sedia Selene, dal nome della divinità greca, personificazione della luna. Nel caso di Danese, invece, fu inizialmente operata la scelta di chiamare con il nome di isole tutti i prodotti del brand: da qui il nome delle isole inglesi per la lampada Falkland, il nome dell' isola italiana Formosa per un calendario perpetuo da parete, il nome dell'isola dell'arcipelago malese Timor per il calendario da tavolo, il nome delle isole Kerguelen, nell'arcipelago dell'Oceano Indiano, per un appendiabiti; per il vaso Pago Pago è stato scelto invece il nome della capitale dell'isola di Tutuila nell'arcipelago delle isole Samoa Americane, utilizzato in questo caso anche per richiamare l'idea progettuale di Enzo Mari, in cui due vasi si uniscono tra loro per formare un unico oggetto, come due sono le parole che formano il nome della città (Annicchiarico 2002c: 88). Anche Memphis annovera numerosi nomi di luoghi esotici (Annicchiarico 2002c: 30): è il caso, ad esempio, del mobile da soggiorno Casablanca e della lampada da tavolo Tahiti.

Nelle strategie di naming per gli oggetti di design si possono anche individuare alcuni campi semantici ricorrenti, come risulta dalle ricerche e dalle mostre realizzate per la Triennale di Milano da Annicchiarico (2002a, 2002b, 2002c), che riconosce la diffusione di tre ambiti semantici in particolare, i nomi di donna, i nomi di animali e i nomi di luoghi, evidenziando i risvolti culturali e simbolici delle denominazioni.

La scelta di nomi di donne - nomi di persona, nomi letterari o mitologici - o di appellativi femminili (come Donna o Lady) può essere legata, come abbiamo visto, a una analogia morfologica (es. Donna), a una indicazione di stile (es. Mirella o Lady), a una dedica (es. Luisa) o a una decisione di marketing aziendale (come nel caso di Artemide). Il ricorso a nomi di donna sembra dipendere, tuttavia, anche da una motivazione di carattere emozionale, come suggeriscono le parole del designer Paolo Rizzato: "creare un oggetto è un po' come concepire una creatura" [... ], "finisce che ti ci affezioni, e che la chiami con un nome di donna, perché ti ispira sentimenti di tenerezza, protezione e intimità" (Annicchiarico 2002: 27). Il nome femminile opera inoltre una sorta di risemantizzazione dell'oggetto: il prodotto di serie viene avvolto da una dimensione comunicativa che condensa una "gamma estremamente ricca di relazioni e di narrazioni possibili” (Annicchiarico 2002: 28).

L'uso dei nomi di animali è un'altra frequente modalità espressiva, come viene confermato anche dall'analisi del nostro corpus. Tra l'animale e l'oggetto si instaura un rapporto di somiglianza per il rimando alla forma o al movimento oppure, in modo meno evidente, in relazione alla fonte di ispirazione formale del progettista, che viene svelata dal richiamo allanimale, come abbiamo 
osservato per i nomi Canguro o Papillon. Ma gli oggetti zoomorfi del design, secondo Annicchiarico (2002b: 29-36), hanno anche un valore simbolico: gli oggetti con il nome di un animale evocano il ricordo della naturalità e "si offrono come surrogati di quell'animalità che non ha (e non può avere) luogo nell'abitazione moderna", con una intenzionalità spesso "ludica e giocosa" nella scelta di denominazione dell'oggetto.

I prodotti con nomi di luoghi sono quelli più numerosi, secondo le ricerche di Annicchiarico, che include in questa categoria i toponimi, come nomi di città o isole, i nomi di ambienti o elementi della natura (es. I sassi, Atollo) e i nomi di elementi architettonici (es. Capitello). Lattribuzione di nomi di luoghi, legata ad analogie formali, a riferimenti ad occasioni d'uso, aspetti stilistici o ricordi, oppure a semplici scelte aziendali (come nel caso di Danese e Memphis), può essere letta, anche in questo caso, in una dimensione simbolica e allusiva. L'ampia varietà dei nomi di luoghi "risponde a una logica precisa" (Annicchiarico 2002c: 31 ), come se permettesse di abbracciare il mondo esterno nella sua pluralità e interezza: gli oggetti evocano così il mondo esterno e questo mondo entra nelle case.

\section{Percorsi per la didattica dell'italiano L2}

I nomi commerciali del settore del design italiano, dai marchionimi aziendali ai nomi dei prodotti, appartengono alla storia e alla cultura italiana e risultano rilevanti anche in una prospettiva glottodidattica, offrendo materiale linguistico e contenuti culturali da utilizzare nella classe di italiano L2.

I marchi aziendali e i nomi di prodotto, insieme ai testi ad esso correlati, ossia quei testi che ne svelano l'origine e il significato, vengono così a costituire testi input di unità di lavoro, intorno a cui ruotano le attività didattiche (Vedovelli 2010).

Si tratta di attività didattiche che possono essere calibrate su diversi livelli di competenza e che possono essere realizzate in diversi contesti di apprendimento, in Italia o all'estero, in particolare con apprendenti giovani e adulti, con un interesse verso la cultura italiana di carattere generico o specifico, come nel caso di studenti in formazione in contesto scolastico o universitario.

I marchionimi aziendali forniscono l'occasione per realizzare percorsi didattici su storiche aziende italiane, che testimoniano la creatività e l'imprenditorialità italiana nella storia e nel mondo, come Alessi, Artemide, Aurora, Kartell, Olivetti, ecc. Al marchionimo può essere abbinata la didattizzazione di testi facilmente reperibili, in genere, nei siti ufficiali delle aziende, attraverso i quali l'apprendente può essere guidato alla scoperta dell'origine del marchio, che esprime la storia e l'immagine dell'azienda. 
I testi della sezione del sito dedicata alla storia della nascita dell'azienda contengono informazioni che consentono, nella maggior parte dei casi, di individuare intuitivamente l'origine del marchionimo, in particolare quando è costituito da un antroponimo, il nome del fondatore o della famiglia del fondatore dell'azienda. In altri casi, quando l'origine dei marchi è legata a relazioni semantiche e analogie di altro genere (come per Tecnica, Flos, Vortice, ecc.), i testi di riferimento possono essere tratti anche da altre fonti o da altre sezioni del sito, come nelle pagine dedicate alla presentazione dell'immagine e dei valori aziendali.

Come attività didattiche si possono realizzare ad esempio:

- attività di comprensione scritta di testi associati ai marchionimi proposti con l'elemento grafico del logo, attraverso tecniche come abbinamento tra marchionimo e testo oppure domande chiuse o aperte;

- attività di produzione orale o scritta sulla storia e l'analisi di marchionimi a partire dalle informazioni presenti nei testi forniti dal docente;

- attività di produzione scritta o orale sulla storia e l'analisi di marchionimi sulla base di una ricerca di informazioni libera o guidata su internet.

I nomi di prodotto, invece, possono offrire materiale linguistico per attività di carattere creativo e ludico basate sulla scoperta delle motivazioni semantiche e dei giochi linguistici legati alle strategie di denominazione. In questo caso i nomi devono essere corredati dai testi descrittivi dei prodotti, reperibili da varie fonti e generi testuali, quali le presentazioni dei prodotti nei siti aziendali o le descrizioni presenti in cataloghi di mostre, riviste online o blog. Dai testi si possono ricavare le informazioni utili per ricostruire le motivazioni alla base della creazione dei nomi, riconoscibili in modo semplice e immediato oppure con un maggiore sforzo interpretativo, a seconda della maggiore o minore trasparenza semantica dei nomi, come abbiamo visto dall'analisi del corpus.

Riportiamo a titolo esemplificativo due testi descrittivi di oggetti precedentemente menzionati. Il primo testo è tratto dal sito dell'azienda Artemide e descrive la lampada Eclisse creata da Vico Magistretti nel 1965, mentre il secondo è presente nell'archivio digitale della Fondazione Aldo Rossi e riporta il pensiero di Aldo Rossi sulla creazione dell'armadio Cabina dell'Elba:

Eclisse è un equilibrio all'avanguardia tra forma e funzione, design e utilità. La base del concetto sta nella sua funzionalità di regolazione dell'intensità della luce attraverso il suo paralume interno rotante che "eclissa" la sorgente luminosa. Infatti, con un involucro esterno fisso e un involucro interno mobile, la lampada può fornire luce diretta o diffusa. (dal sito di Artemide)

Questi armadi, o costruzioni, risalgono agli anni passati. [... $]$

In realtà mi ero semplicemente accorto del carattere particolare e universale delle cabine poste sulle spiagge. Non solo dell'Elba. Il problema non è quello 
di vedere ma di guardare fino ad appropriarsi dell'immagine, e attraverso l'immagine della cosa.

Così ho incontrato migliaia di cabine, dalle spiagge del Mediterraneo, alla California, all'Argentina.

E con piacere ho visto ripetizioni della mia cabina, ma non le considero copie, anzi è come rivedere le cose e riscoprirle con sorpresa.

La cabina è una piccola casa: è la riduzione della casa, è l'idea della casa. (www.fondazionealdorossi.org/)

Oltre ai nomi di prodotti presenti nel nostro corpus, dai siti aziendali o dai cataloghi di mostre si possono poi anche reperire, evidentemente, numerosi altri nomi di oggetti, con i relativi testi descrittivi, che mostrano la creatività nella denominazione degli oggetti presente nel mondo del design italiano.

Insieme ai nomi in italiano, inoltre, possono essere utilizzati anche nomi in lingue diverse dall'italiano, che possono fornire lo spunto per riflessioni interlinguistiche e interculturali, come per esempio nel caso della poltrona Blow, della Spaghetti Chair o del tavolo Kyoto.

Le attività didattiche che possono essere svolte con i nomi di prodotti sono ad esempio:

- attività di comprensione scritta di testi associati ai nomi dei prodotti e all'immagine relativa, attraverso tecniche come abbinamento tra nome di prodotto e testo oppure domande chiuse o aperte;

- attività di produzione orale o scritta di testi sull'origine e il significato di nomi di prodotto a partire dalla rielaborazione delle informazioni presenti nei testi forniti dal docente;

- attività di produzione orale o scritta di testi attraverso la tecnica della transcodificazione, sulla base del riconoscimento di possibili relazioni tra il nome attribuito all'oggetto e l'immagine dell'oggetto stesso, partendo dai nomi più descrittivi e referenziali per arrivare alle associazioni di carattere metaforico;

- attività di produzione orale o scritta sull'origine e il significato di nomi di prodotto sulla base di una ricerca di informazioni libera o guidata su internet.

\section{Riflessioni conclusive}

L'indagine condotta sui nomi commerciali raccolti nel corpus ha evidenziato il ruolo del settore del design italiano nella diffusione dell'italianità nel mondo, attraverso marchi e prodotti altamente rappresentativi del valore del made in Italy, non solo a livello economico ma anche culturale. 
Dall'analisi linguistica e semantica dei marchionimi è emersa un'ampia creatività onomastica, in particolare nel caso della denominazione dei prodotti, in cui la lingua italiana offre materiale per la creazione di nomi originali ed evocativi, insieme ad altre lingue che evidenziano la dimensione internazionale del settore.

In una prospettiva glottodidattica la conoscenza dei nomi commerciali, che è parte della cultura legata alla lingua, rappresenta un campo di interesse e di sviluppo per la didattica delle lingue. Il design, in quanto esempio di un settore di rilevanza nella storia economica e culturale italiana, può essere dunque efficacemente integrato nella didattica dell' italiano L2, anche attraverso percorsi di scoperta e analisi dei nomi commerciali.

I marchionimi del design italiano e i testi ad essi correlati, da cui trarre le motivazioni all'origine della creazione dei nomi, possono così diventare materiali da didattizzare per l'insegnamento e l'apprendimento dell'italiano L2: con i nomi aziendali, a partire dalla ricostruzione della loro origine, si possono realizzare percorsi didattici sulla storia e i valori di grandi aziende italiane; con i nomi dei prodotti si possono progettare attività per scoprire le caratteristiche linguistiche e le motivazioni semantiche cui i nomi rimandano, come le relazioni con gli elementi formali dell'oggetto, il suo uso, le sue caratteristiche distintive $o$ aspetti stilistici.

Il ricorso ai nomi commerciali nella didattica dell'italiano L2 può favorire, dunque, la motivazione nell'apprendimento linguistico, attraverso la realizzazione di attività che stimolano la partecipazione attiva dell'apprendente. Nella scoperta delle motivazioni soggiacenti alla creazione dei nomi, l'apprendente viene coinvolto in attività che rappresentano una sfida interpretativa - una sfida con sè stesso o con altri studenti - e sollecitano la capacità di risoluzione dei problemi attraverso la lingua in una dimensione ludica: ciò contribuisce a incrementare il piacere, il divertimento e l'interesse, su cui si fonda la motivazione nell'apprendimento.

\section{Bibliografia}

Alessi, Chiara (2014). Dopo gli anni Zero. Il nuovo design italiano, Roma-Bari, Laterza.

Annicchiarico, Silvana (2002). "Quando gli oggetti hanno un nome di donna”, [in] Silvana Annicchiarico (a c. di), Non sono una signora. Quando gli oggetti hanno un nome di donna, Milano, Edizioni Charta, pp. 21-33.

Annicchiarico, Silvana (a c. di) (2002a). Non sono una signora. Quando gli oggetti hanno un nome di donna, Milano, Edizioni Charta.

Annicchiarico, Silvana (a c. di) (2002b). Animal house. Quando gli oggetti hanno forme e nomi di animali, Milano, Edizioni Charta.

Annicchiarico, Silvana (a c. di) (2002c). Il mondo in una stanza. Quando gli oggetti hanno nomi di luoghi, Milano, Edizioni Charta. 
Annicchiarico, Silvana (2007). 100 oggetti del design italiano. Collezione permanente del design italiano. La Triennale di Milano, Roma, Gangemi Editore.

Bagna, Carla e Barni, Monica (2007). "La lingua italiana nella comunicazione pubblica/sociale planetaria”, Studi italiani di linguistica teorica e applicata, XXXVI, pp. 529-553.

Bassetti, Pietro (2010). "Multilinguismo, pluriappartenenza e italicità", [in] Nicoletta Maraschio, Domenico De Martino e Giulia Stanchina (a c. di), Esperienze di multilinguismo in atto, Firenze, Accademia della Crusca, pp. 147-152.

Bassetti, Pietro (2015). "Svegliamoci italici! Manifesto per un futuro glocal”, [in] Raffaella Bombi e Vincenzo Orioles (a c. di), Italiani nel mondo. Una Expo permanente della lingua e della cucina italiana, Udine, Forum, pp. 59- 62.

Bombi, Raffaella (2014). "Italicità, food e slow food. Una nuova dimensione culturale ed espressiva”, [in] Raffaella Bombi e Vincenzo Orioles (a c. di), Essere italiani nel mondo globale oggi. Riscoprire l'appartenenza, Udine, Forum, pp. 65-76.

Bombi, Raffaella e Orioles, Vincenzo (2011). "Scenari dell'italianità nel mondo. Da oriundi a italici, con il valore aggiunto della doppia appartenenza”, [in] Raffaella Bombi e Vincenzo Orioles (a c. di), Nuovi valori dell'italianità nel mondo. Tra identità e imprenditorialità, Udine, Forum, pp. 11-29.

Bombi, Raffaella e Orioles, Vincenzo (2014). "Dalla ricerca alla formazione. Come veicolare i valori emergenti dell'italicità”, Studi Italiani di Linguistica Teorica e Applicata, 2, pp. 305-323.

Caffarelli, Enzo (2011). Nomi commerciali, [in] Enciclopedia dell'Italiano, Roma, Istituto dell'Enciclopedia Italiana, www.treccani.it/enciclopedia/nomi-commerciali_(Enciclopedia-dell'Italiano)/ [20/05/2020].

Cartago, Gabriella (2016). "Italian design" e disegno italiano: un bilancio linguistico attraverso il tempo", [in] Paolo D’Achille e Giuseppe Patota (a c. di), L'italiano e la creatività. Marchi e costumi, moda e design, Firenze, Accademia della Crusca, pp. 11-22.

Cotticelli Kurras, Paola (2008). "La struttura morfologica dei marchionimi italiani nel XX secolo (fino agli anni '80)", [in] Maria Giovanna Arcamone, Donatella Bremer, Davide De Camilli e Bruno Porcelli (a c. di), I nomi nel tempo e nello spazio. XXII Congresso Internazionale di Scienze Onomastiche (ICOS), Edizioni ETS, Pisa, pp. 695-709.

Cotticelli Kurras, Paola (2011). "Larchivio dei marchi della CCIAA di Verona: strategie linguistiche e sinergie di marketing", [in] Marta Ugolini (a c. di), Branding Verona. Un viaggio tra protagonisti e strumenti delle politiche di marca delle imprese veronesi, Verona, Cierre, pp. 123-149.

Cotticelli Kurras, Paola (2012). "Dal Drivecaffè alla Snackerina ... Neoformazioni dei marchionimi italiani: tendenze a cavallo del secolo", [in] Paola Cotticelli Kurras e Elke Ronneberger-Sibold (a c. di), Il linguaggio della pubblicità italiano e tedesco: teoria e prassi - Italienische und deutsche Werbesprache: Theorie und Praxis, Alessandria, Edizioni dell'Orso, pp. 85-103.

Coveri, Lorenzo, Finessi Simona e Bigliatti, Cristina (2016). "Tracce di lingua italiana del design”, [in] Paolo D’Achille e Giuseppe Patota (a c. di), L'italiano e la creatività. Marchi e costumi, moda e design, Firenze, Accademia della Crusca, pp. 23-28.

De Mauro, Tullio, Vedovelli, Massimo, Barni, Monica e Miraglia, Lorenzo (2002). Italiano 2000. I pubblici e le motivazioni dell'italiano diffuso fra stranieri, Roma, Bulzoni. 
Dellapiana, Elena e Siekiera, Anna Maria (2015). “Come scrivono i designer: note di letture comparate per una linguistica disciplinare”, AIS/Design. Storia e Ricerche, 6, www.aisdesign. org/aisd/come-scrivono-i-designer-note-di-letture-comparate-per-una-linguisticadisciplinare [20/05/2020].

Dellapiana, Elena e Siekiera, Anna Maria (2017). "Il design italiano. Litaliano del design”, Nuova Corvina, 12, pp. 131-142.

Frosini, Giovanna (2012). "La cucina degli italiani. Tradizione e lingua dell'Italia al mondo", [in] Giada Mattarucco (a c. di), Italiano per il mondo. Banca, commerci, cultura, arti, tradizioni, Firenze, Accademia della Crusca, pp. 85-107.

Galisson, Robert (1988). "Culture et lexiculture partagées: les mots comme lieux d’observation des faits culturels”, Études de Linguistique Appliquée, 69, pp. 74-90.

Galisson, Robert e André, Jean-Claude (1998). Dictionnaire de noms de marques courants. Essai de lexiculture ordinaire, Paris, Didier Erudition.

Gałkowski, Artur (2010). "Problemi di terminologia onomastica. Contributi per un dibattito", Rivista Italiana di Onomastica, XVI, 2, pp. 604-624.

Gałkowski, Artur (2011). "Dalla Fiat alla Lavazza con una sosta dolce alla Nutella..." La conoscenza dei nomi commerciali come elemento della competenza linguistica e interculturale in italiano L2", Italica Wratislaviensia, 2, pp. 79-93.

Gałkowski, Artur (2015). "Il mondo creativo dei nomi propri e la didattica dell'italiano come lingua straniera”, [in] Maria Załęska (a c. di), L'italiano insegnato creativamente, Warszawa, Ka-tedra Italianistyki - Uniwersytet Warszawski, pp. 77-113.

Gałkowski, Artur (2016). "La (non) convenzionalità della crematonimia storica e contemporanea", Rivista Italiana di Onomastica, XXII, 1, pp. 111-124.

Guizzi, Giulio (2015) Pulizia igienica e sanificazione. La sporca storia del pulito, Milano, Edizioni LSWR.

Orioles, Vincenzo (2014). "Verso nuove categorizzazioni dei contesti migratori. Dall'italianità all'italicità, [in] Raffaella Bombi e Vincenzo Orioles (a c. di), Essere italiani nel mondo globale oggi. Riscoprire l'appartenenza, Udine, Forum, pp. 109-116.

Orioles, Vincenzo (2015). "Sul costrutto di italicità. In margine alla presentazione di Svegliamoci italici! di Piero Bassetti”, [in] Raffaella Bombi e Vincenzo Orioles (a c. di), Italiani nel mondo. Una Expo permanente della lingua e della cucina italiana, Udine, Forum, pp. 63-69.

Papa, Elena (2015). "Lo stile Olivetti, dalla macchina al Logos", [in] Oliviu Felecan (a c. di), Name and Naming. Conventional/Unconventional in Onomastics. Proceedings of ICONN3, Baia Mare 1-3 settembre 2015, Editura Argonaut, Cluj-Napoca, pp. 773-785, www. diacronia.ro/ro/indexing/details/V1984/pdf [20/05/2020].

Piva, Antonio e Cao, Elena (a c. di) (2009). Vittoriano Viganò. A come Asimmetria, Roma, Gangemi editore.

Roić, Sergej (a c. di) (2006). Globus et Locus. Il percorso dell'italicità, Lugano-Milano, Giampiero Casagrande Editore.

Sala, Nicoletta e Sala, Massimo (2005). Geometrie del design. Forme e materiali per il progetto, Milano, Franco Angeli.

Sergio, Giuseppe (2016). "Italianismi di moda nelle lingue del mondo", [in] Paolo D’Achille e Giuseppe Patota (a c. di), L'italiano e la creatività. Marchi e costumi, moda e design, Firenze, Accademia della Crusca, pp. 55-68. 
Turchetta, Barbara e Vedovelli, Massimo (a c. di) (2018). Lo spazio linguistico italiano globale: il caso dell'Ontario, Pisa, Pacini.

Vedovelli, Massimo (2005). "L'italiano nel mondo da lingua straniera a lingua identitaria: il caso 'freddoccino', Studi italiani di linguistica teorica e applicata, XXXIV, 3, pp. 585-609. Vedovelli, Massimo (2010). Guida all'italiano per stranieri. Dal Quadro comune europeo per le lingue alla Sfida salutare, Roma, Carocci.

Vedovelli, Massimo (2011). "Lingua ed emigrazione italiana nel mondo. Per uno spazio linguistico italiano globale”, [in] Raffaella Bombi e Vincenzo Orioles (a c. di), Nuovi valori dell'italianità nel mondo. Tra identità e imprenditorialità, Udine, Forum, pp. 33-41.

Vercelloni, Matteo (2014). Breve storia del design italiano, Roma, Carocci. 